survival rates at CCHE were inferior; $79.9 \%$ for ALL; $53.8 \%$ for AML; $56.5 \%$ for Neuroblastoma; $56.4 \%$ for Hepatoblastoma; $49.1 \%$ for Osteosarcoma. Trends in age-adjusted mortality-rates will presented.

Conclusions Studying 5-year survival in childhood cancer health outcomes at CCHE would help generate real-world evidence about those having inferior outcomes and identify priority areas that need future improvements. Making better use of the evidence generated at CCHE would enhance real-world practice through making informed decisions that are adapted to a local context setting- CCHE.

\section{ACHIEVING EVIDENCE INTEROPERABILITY IN THE COMPUTER AGE: SETTING EVIDENCE ON FHIR}

\footnotetext{
1,2Brian Alper, ${ }^{1,3}$ Martin Mayer, ${ }^{1}$ Khalid Shahin, ${ }^{4}$ Joshua Richardson, ${ }^{5}$ Lisa Schilling, ${ }^{6,7}$ Mario Tristan, ${ }^{6}$ Niels Salas. ${ }^{1}$ EBSCO Health, Ipswich, MA, USA; ${ }^{2}$ University of MissouriColumbia School of Medicine, Columbia, MO, USA; ${ }^{3}$ Cone Health, Greensboro, NC, USA; ${ }^{4}$ RTI International, Chicago, USA; ${ }^{5}$ University of Colorado, Aurora, USA; ${ }^{6} \mathrm{HCAl}$, San Jose, Costa Rica; ${ }^{7}$ Cochrane Central America and Spanish-speaking Caribbean, San Jose, Costa Rica
}

\subsection{6/bmjebm-2019-EBMLive.28}

Objectives Evidence-based practice requires the use of research results to inform care. Computers can add capacity for evidence-based practice by making the information from research results, appraisals, and summarizations searchable and re-usable without labor-intensive manual screening and repetition of data entry. Such interoperability can be achieved by establishing universal standards for data exchange for communicating evidence concepts in machine-interpretable formats.

Method Health Level 7 (HL7) is a standards development organization that has developed a standard for electronic exchange of healthcare information called Fast Healthcare Interoperability Resources (FHIR). We are using the HL7 standards development methodology to extend FHIR to create an Evidence Resource for exchanging descriptive, statistical and certainty concepts related to evidence.

Results The FHIR Resources for Evidence-Based Medicine Knowledge Assets (EBMonFHIR) project is in active development with a substantial coalition of international organizations and coordination with other standards development groups. The Statistic Resource currently supports explicit descriptions of the populations and subgroups (exposureBackground elements), interventions or exposures and comparators (exposureVariant elements), the outcomes (measuredVariable elements), and for each statistic the sample size, the value with unit of measure, the precision estimate, the $\mathrm{p}$ value, and the certainty of the statistic. The project website (http://wiki.hl7.org/index. php?title $=$ EBMonFHIR) includes multiple examples and information on how to participate.

Conclusions Working together we can achieve interoperability for evidence in the electronic era to realize the technological breakthroughs we see in other domains such as navigation support. A common information architecture will also facilitate the harmonization of 'Real World Evidence' and 'Evidence Based Medicine' which collectively represent clear understanding of evidence and its certainty, regardless of evidence source. Extending the solutions achieving interoperability for healthcare services provide a means to not only solve this challenge for the Evidence Ecosystem but also to keep it well connected with healthcare services delivery.

\section{THE USE OF EVIDENCE IN HUMANITARIAN RESPONSE DECISION MAKING}

${ }^{1}$ Dell Saulnier, ${ }^{2}$ Claire Allen, ${ }^{1}$ Anneli Eriksson, ${ }^{2}$ Ben Heaven Taylor. ${ }^{1}$ Karolinska Institutet, Stockholm, Sweden; ${ }^{2}$ Evidence Aid, London, UK

\subsection{6/bmjebm-2019-EBMLive.29}

Objectives The need to use evidence in humanitarian settings is recognized yet utilising that evidence to make decisions about humanitarian response remains a challenge. The objective of the study was to identify how, when, and why decision makers in humanitarian response use scientific, peer-reviewed evidence to make decisions.

Method An online cross-sectional survey of fifteen open- and closed-ended questions on demographics, experience and role in humanitarian response was developed by Evidence Aid (EA) and Karolinska Institutet (KI). The online survey was available on the EA website from 20 August-15 October 2018. Participants were self-selected, recruited through social medial channels and mailing lists of EA and KI. All respondents and responses were anonymized. Responses were analyzed with descriptive statistics and content analysis.

Results Forty-seven people responded, primarily working in Europe or North America with roles of humanitarian response director/manager, independent consultant, or policy maker. Personal assessment of the quality of information, trust in the source, and information that was contextually relevant or based on field experience were factors for deciding whether information should be considered evidence. Reasons for using evidence when making decisions included adhering to good practice to maximize impact and effectiveness of aid, reassurance that the right decisions were being made, personal or organizational values, and using evidence as a tool to protect beneficiaries and organizations from poor quality decisions and program content.

Conclusions Using evidence for decision making was common practice during the process of designing implementing and evaluating humanitarian response content, yet reasons for use varied. The importance of evidence developed and validated from field experience and trust in the source reported by this sample suggests that strengthening collaborative efforts between decision makers and evidence generators could be one approach to improve evidence and evidence use in humanitarian response.

\section{DECISION AIDS FOR ORGANIZED CANCER SCREENING IN GERMANY}

Dennis Fechtelpeter, Roland Büchter, Milly Schröer-Günther, Regina Meurer, Beate Zschorlich, Klaus Koch. Institute for Quality and Efficiency in Health Care (IQWG), Cologne, Germany

\subsection{6/bmjebm-2019-EBMLive.30}

Objectives Germany has organized programs for breast, colorectal and cervical cancer screening. The target audience receives regular letters that are accompanied by a decision aid. Besides general information about the screening programmes, the decision aids include description of the advantages and disadvantages of screening to support informed choice. The Institute for Quality and Efficiency in Health Care (IQWiG) was commissioned to produce the cover letters and the decision aids. The development process and the user testing results will be presented. 